\title{
Discussion on Schumann Resonance Measurement and Data Processing
}

\author{
Bingxia Cao, Jinghong Xue, Hongjuan Zhou \\ School of Information and Electrical Engineering \\ Harbin Institute of Technology, HIT \\ Weihai, China \\ jchdd1980@163.com, xuejing_h@126.com, \\ Hongjue_zhou@163.com
}

\author{
Jingjing Zhang \\ Shandong Astronautics Electronic Technology Institute \\ Yantai, China \\ summer2002198320@163.com
}

\begin{abstract}
Schumann Resonance is the natural atmosphere noise in ELF band. The measurement and data processing are discussed in the paper. The composition of the equipment is described. SR data processing methods are detailed, including FFT method, periodogram, SVD filtering and curve fitting. The results by different data processing methods are compared. And strengths and weaknesses illustrated.
\end{abstract}

Keywords-Schumann Resonance; data processing; periodogram; SVD; curve fitting

\section{INTRODUCTION}

The Earth-ionosphere cavity, composed of a thin insulator (the atmosphere) confined between two spherical conductors (the Earth and the ionosphere), provides the natural framework for both the DC and AC global circuits[1, 2].

The propagation of low frequency electromagnetic waves within the cavity was first studied early in 1950s. The cavity is excited with broadband electromagnetic sources. The average equatorial circumference is approximately equal to an integral number of wavelengths of the electromagnetic waves[3]. So a resonant state develop. The natural phenomenon is known as Schumann Resonance (SR) which is first proposed by Schumann. And it was subsequently observed by Balser and Wagner[4]. They have peak frequencies for the first four modesat 7.8, 14, 20 and $26 \mathrm{~Hz}[4]$.

The upper boundary of Schumann resonance cavity is the bottom of the ionosphere. That is the transition region from the atmospheric to the ionosphere. Research on SR is important to reveal many geophysical phenomena at the bottom of the ionosphere[2, 5]. The SR measurement can provide enough information of the lightning and thunderstorm activity. SR also can act as the global tropical thermometer. In recent years, researches on the Schumann resonance for the prediction of short impending earthquake prediction[6-9] are reported. SR as a new electromagnetic precursor of earthquakes, has the advantages of relatively strong anti-interference ability[3, 10-12], so it has been given widespread attentions.

The majority of lightning flashes cannot stand out above all other events. And the electromagnetic fields superimpose to produce the quasi-steady 'background' resonances. The phase information for the integrated activity cannot be obtained. So the observations are usually recorded as power spectra of electric and magnetic fields.
The electromagnetic fields are usually concluded three components, vertical electric, north-south magnetic and east-west magnetic[10, 13].

The ELF atmospheric background noise measurement instrument and the usual data analysis methods used is introduced in the paper.

\section{MEASUREMENT EQUIPMENT OF SCHUMANN RESONANCE}

The most popular Schumann resonance observation method is to use three extremely low frequency (ELF) sensors, including East-West and North-South direction for magnetic field and a vertical one for electric field[7][14, 15]. The schematic block diagram of a typical SR measurement equipment is shown in Fig.1. There are three sensors, or called antenna, two perpendicular horizontal magnetic antennas, a vertical electric ball antenna. The magnetic antennas (MA) are induction coil son an amorphous ferromagnetic core. The receiving equipment comprises three channels. Each channel carries a pre-amplifier, scaling amplifier and low pass filter (LPF). Signals from the three channels are sent into a computer for data processing after sampled in A/D converter.

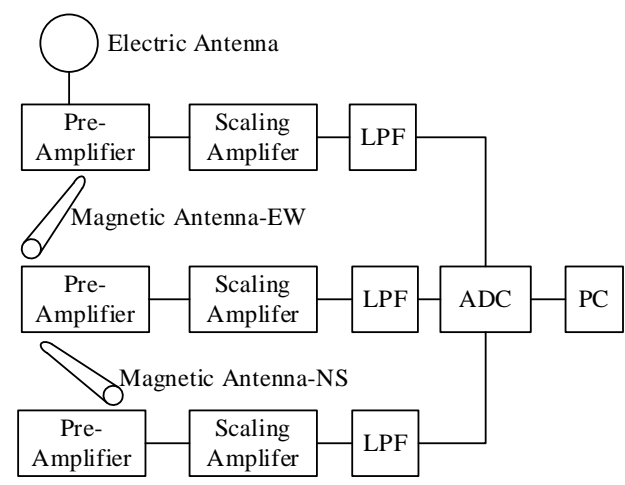

Fig. 1. The schematic block diagram of SR measuring instrument

In recent years, Japanese researchers have found seismic activity may be associated with abnormalities of the Schumann resonances in the ELF atmospheric background noise observing[11,12]. Researchers have conducted a comparative study of earthquakes and the statistical properties of seismic activity and get some of the Schumann resonance abnormal 
relationship, but also some still unable to explain the phenomenon [11,13 ] .

Some such equipment comprises three magnetic induction antennas for only magnetic field observing. Three magnetic sensors can simultaneously measure the 3 axial magnetic field components: South-North (SN) direction component, the East-West (EW) direction and vertical components (Vertical).The sensor signal is pre-amplified firstly and then filtered by a low pass filter. After a long cable travelling, it will be amplified by the main amplifier and then be sampled by the A/D converter. The data in this paper is provided by such equipment.The sampling rate is $100 \mathrm{~Hz}$.

\section{DATA PROCESSING OF SCHUMANN RESONANCE}

\section{A. Periodogram Method}

Schumann Resonance acts as a natural background atmosphere noise. The SNR is really very low. And the sampled SR signal in time domain is completely submerged as seen in Fig.2. So the first task of data processing is to extract the SR signal from the wideband noise.

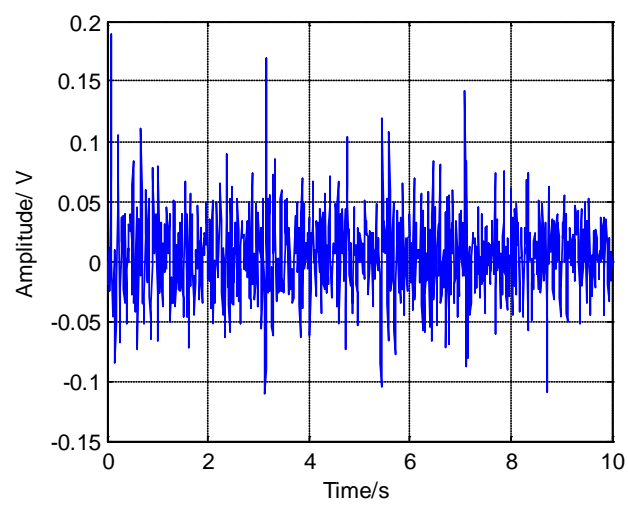

Fig. 2. SR signal in time domain

The spectrum peaks in frequency domain are relatively stable. So the parameters of SR are studied in frequency domain. FFT is carried out first for the sampled data. But because of the serious noise, frequency spectrums are in clutter as seen in Fig.3. It is difficult to obtain high precision parameters of SR spectral peaks under such conditions.

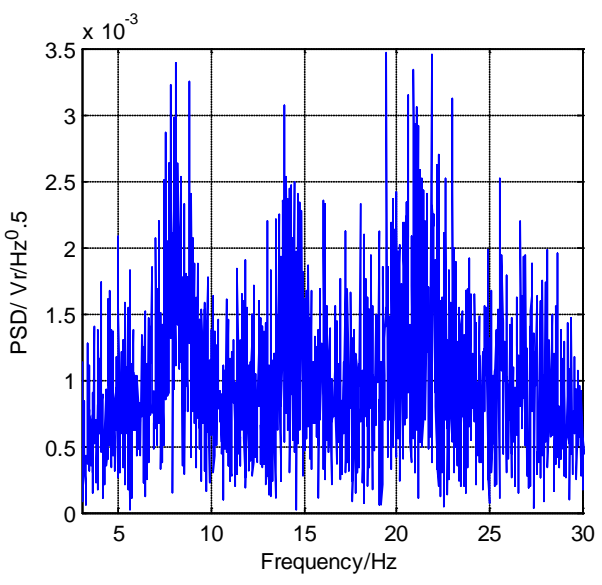

Fig. 3. SR spectrums by FFT

In order to get a smooth curve, periodogram method is used here. The curves in Fig. 4 (a) and (b) separately represent the dealing results of 5 minute and 1 hour data. And the FFT resolution is also $0.1 \mathrm{~Hz}$. The longer the accumulation time, the clearer the SR spectrum curve. Fig. 4 (a) is the result for 5 minutes accumulation. There are some spikes interference and the location and amplitude of the peaks are not easy to distinguish. But in Fig.4(b), the spectrum curve gets very smooth, and the shape of the peaks also gets better. If the first order of SR peak is statistically analyzed from the result in Fig.4(a), the position is about $7.6 \mathrm{~Hz}$ and the amplitude is about 1.26e-5. But in Fig.4(b), those should be $7.9 \mathrm{~Hz}$ and $1.54 \mathrm{e}-5$. There are also similar problems for other peaks. Long average time can bring smaller impact of the interference and more accurate statistical results. But the real time performance get worse. So a suitable accumulation time length is very important. Usually the value is taken for an hour or half an hour. Fig. 5 is the result after data smoothing, with half an hour accumulation.
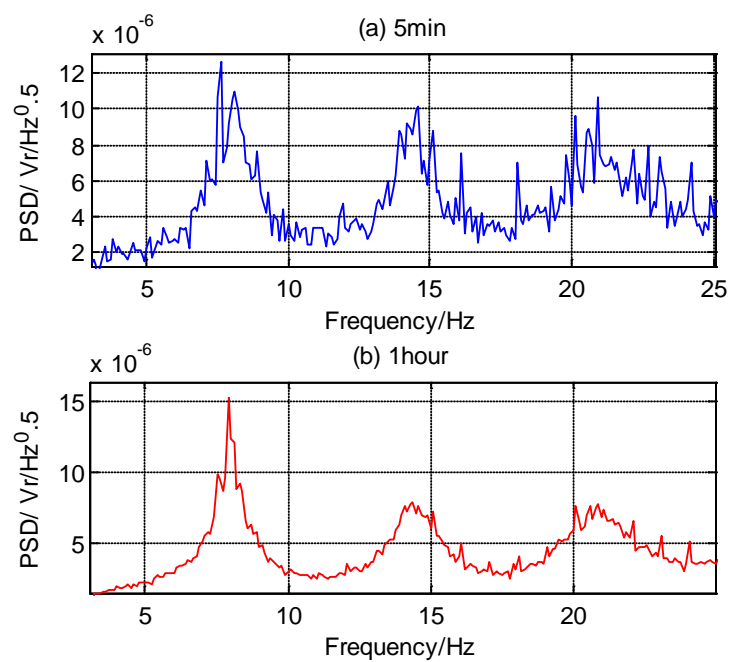

Fig. 4. SR spectrums by periodogram 


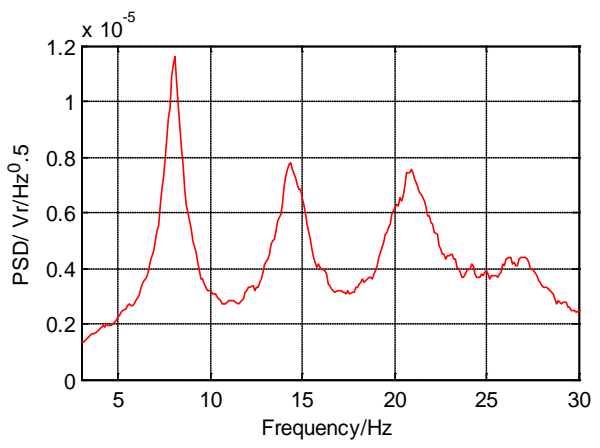

Fig. 5. SR spectrums after smoothing

\section{B. Singular Value Decomposition}

Background noise can effectively be suppressed by singular value decomposition (SVD) filtering method.The SVD filtering method is usually used for image background suppression and de-noising for its good numerical robustness and adaptability.

SR can be regarded as a kind of special ELF frequency atmospheric background noise. So it is very important to choose the appropriate number of the singular value for the SVD algorithm to obtain better data processing result. SVD Matrix is constructed here based on the time domain SR data of an hour. There are 360 singular values. And the result by selecting the first 50 singular values for reconstruction is as shown in Fig.6. The substrate noise voltage of SR spectrum is reduced overall by SVD filtering compared to periodogram method. The amplitude of the 1st SR spectrum peak keeps nearly the same value in the two processing method. But the other peaks significantly get decreased. The SNR of the signal is very important for the parameter statistics.

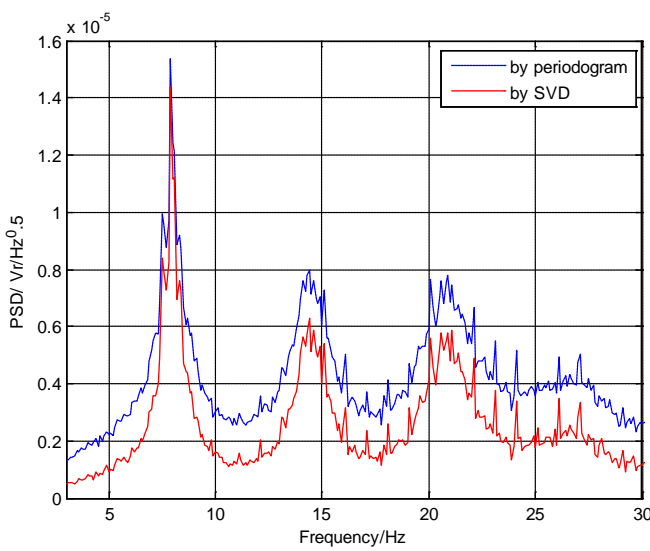

Fig. 6. SR spectrums by SVD filtering

SNR value of SR can be easily obtained from the data in Fig. 7. Obviously, SNR of SR peaks by SVD filtering is higher than that by the periodogram accumulation. For the 1st SR peak, SNR is about $14 \mathrm{~dB}$ by SVD while $8 \mathrm{~dB}$ by periodogram. For the 2nd and 3rd SR peak, the value is improved from $5 \mathrm{~dB}$ to $8 \mathrm{~dB}$. At the same time, the spectrum peaks become narrowing. All these are beneficial to the parameter extraction and reduce the influence of the noise on the parameter statistics.
But in Fig.7, it can be seen that there are some obvious spikes on higher order spectrum by the SVD method.

Since the SVD can only remove the random background noise, it is invalid to the interference at some certain frequencies.

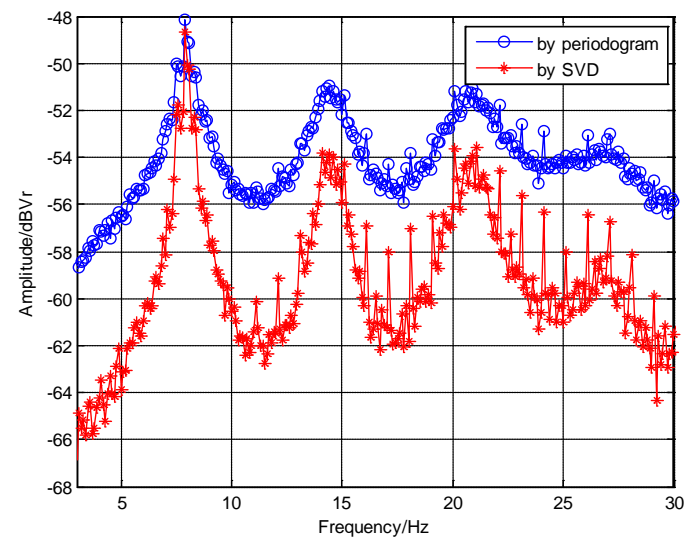

Fig. 7. SNR of SR spectrums by two methods

\section{Curve Fitting}

Curve fitting is also a common method in SR data processing. Fig. 8 is the result by FFT and spectrum estimation of harmonics based on prony algorithm. The peak positons by the prony spectrum estimation is consistent with the actual data. And the fitting curve is clear. But for the fitting result, the relative amplitude of the peaks is changed. The higher order spectrum peaks are notably decreased compared to the 1st order. So this method may be suitable for the peak frequency statistics but not for amplitude statistics.

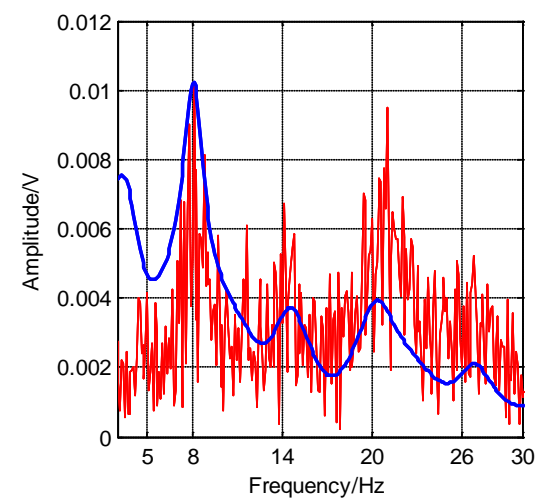

Fig. 8. The co-polarization and cross-polarization phase of linear polarized horn antenna.

\section{CONCLUSION}

The most popular Schumann resonance measurement equipment consists of three channels, for East-West and North-South magnetic field and vertical electric field. Some special observation needs the vertical magnetic field.

There are different methods for SR data processing: 
- $\quad$ Pure FFT method is easy to get the spectrumin cluster. The background noise need reduced for further for parameter statistics.

- Periodogram method need some time for accumulation to reduce the background noise.

- $\quad$ Random background noise can be reduced by SVD algorithm and SNR are enhanced obviously. It is invalid to the interference at some certain frequencies.

- $\quad$ SR spectrum can be obtained from less data by curve fitting. The method may be more suitable for the peak frequency statistics.

\section{ACKNOWLEDGMENT}

This work was supported by the Young Scientists Fund of the National Natural Science Foundation of China (Grant No. 61301014) and the Natural Science Foundation of Shandong of China (Grant No. ZR2013FQ017).

\section{REFERENCES}

[1] A.P.Nickolaenko, "Modern aspects of Schumann resonance studies," Journal of Atmospheric and Solar-Terrestrial Physics, 1997,pp. 805-816.

[2] E. Williams and E. Mareev, "Recent progress on the global electrical circuit,” Atmospheric Research, 2014, pp. 208-227.

[3] E.R.Williams, "Electricityin the Atmosphere-Global Electrical Circuit," Encyclopedia of Atmospheric Sciences (Second Edition),Oxford:Academic Press,2015,pp. 1-8.

[4] M.Balserand C.A. Wagner, "Observations of Earth-ionosphere cavity resonances,” Nature, 1960,pp. 638-641.

[5] V.V.Surkovet al., "Estimation of lightning and sprite parameters based on observation of sprite-producing lightning power spectra,” Journal of Atmospheric and Solar-Terrestrial Physics, 2010, pp. 448-456.
[6] M.Hayakawaet al., "Interpretation in terms of gyrotropic waves of Schumann-resonance-like line emissions observed at Nakatsugawa in possible association with nearby Japanese earthquakes,” Journal of Atmospheric and Solar-Terrestrial Physics, 2010, pp. 1292-1298.

[7] K.Ohta, N. Watanabe, and M. Hayakawa, "Survey of anomalous Schumann resonance phenomena observed in Japan,in possible association with earthquakes in Taiwan,” Physics and Chemistry of the Earth, Parts A/B/C, 2006, pp. 397-402.

[8] K.Ohta, J. Izutsu, and M. Hayakawa, "Anomalous excitation of Schumann resonances and additional anomalous resonances before the 2004 Mid-Niigata prefecture earthquake and the 2007 Noto Hantou Earthquake," Physics and Chemistry of the Earth, Parts A/B/C, 2009, pp. 441-448.

[9] Qi, S., et al., "Resonance effect existence or not for landslides triggered by 2008 Wenchuan earthquake: A reply to the comment by Drs. Xu Chong and Xu Xiwei,” Engineering Geology, 2012, pp. 128-130.

[10] G.Tatsis, et al., "A prototype data acquisition and processing system for Schumann resonance measurements,” Journal of Atmospheric and Solar-Terrestrial Physics, 2015, pp. 152-160.

[11] X.Y.Ouyang, et al., "Variability of Schumann resonance parameters observed at low latitude stations in China,” Advances in Space Research, 2015, pp. 1389-1399.

[12] H.Zhou, et al., "Diurnal and seasonal variations in the Schumann resonance parameters observed at Chinese observatories,” Journal of Atmospheric and Solar-Terrestrial Physics, 2013, pp. 86-96.

[13] G.G.Belyaev, et al., "Schumann resonances observed using Poynting vector spectra,” Journal of Atmospheric and Solar-Terrestrial Physics, 1999, pp. 751-763.

[14] A.V.Shvets, "A technique for reconstruction of global lightning distance profile from background Schumann resonance signal,” Journal of Atmospheric and Solar-Terrestrial Physics, 2001, pp. 1061-1074.

[15] V.G.Bezrodny, "Magnetic polarization of the Schumann resonances: An asymptotic theory,” Journal of Atmospheric and Solar-Terrestrial Physics, 2007, pp. 995-1008. 